\title{
Instabilities in Binary Mixtures of One-Dimensional Quantum Degenerate Gases
}

\author{
M. A. Cazalilla ${ }^{1}$ and A. F. $\mathrm{Ho}^{2,1}$ \\ ${ }^{1}$ Donostia International Physics Center (DIPC), Manuel de Lardizabal 4, 20018-Donostia, Spain. \\ ${ }^{2}$ School of Physics and Astronomy, The University of Birmingham, Edgbaston, Birmingham B15 2TT, UK.
}

\begin{abstract}
We show that one-dimensional binary mixtures of bosons or of a boson and a spin-polarized fermion are Luttinger liquids with the following instabilities: i) For different particle densities, strong attraction between the mixture components leads to collapse, while strong repulsion leads to demixing, ii) For a low-density mixture of two gases of impenetrable bosons (or a spin-polarized fermion and an impenetrable boson) of equal densities, the system develops a gap and exhibits enhanced pairing fluctuations when there is attraction between the components. In the boson-fermion mixture, the pairing fluctuations occur at finite momentum. Our conclusions apply to mixtures both on the continuum and on optical lattices away from integer or fractional commensurability.
\end{abstract}

PACS numbers: 3.75.Kk, 05.30.-d,67.60.-g

The exploration of properties of matter at increasingly lower temperatures and densities has yielded many surprises. The achievement of Bose-Einstein condensation (BEC) in dilute gases of ultracold atoms [1] is one beautiful example. Ever since the observation of BEC, one line of current experimental (and theoretical) studies is on cold gases in very elongated atomic traps. In effectively one-dimensional (1D) systems strong phase fluctuations can occur. These systems are interesting for both large and small $\gamma$ values, where $\gamma=M g / \hbar^{2} \rho_{0}$ is the dimensionless coupling ratio, $g$ is the coupling characterizing the interaction between the atoms in $1 \mathrm{D}, M$ the atom mass, and $\rho_{0}$ the particle density. For $\gamma \sim 1$, a single-component 1D Bose fluid is a quasicondensate with a fluctuating phase but strongly suppressed density fluctuations 22]. Large $\gamma$ leads to a crossover to the Tonks-Giradeau (TG) regime [2]: the bosons become impenetrable, and resemble noninteracting fermions [4]. The low-energy properties in both regimes can be well described by the Luttinger liquid concept $[5,[6]$.

These aforementioned regimes make up the state space of the single-component system of 1D repulsive bosons. It may therefore seem surprising that adding a second component to form a mixture of two types of bosons $(B+$ $B$ ) or of a boson plus a spin-polarized fermion $(B+F)$ can lead to a richer phase diagram. In this Letter, we show that such binary mixtures are Luttinger liquids which can become unstable to gap-opening in one branch of the excitation spectrum, or to demixing, or to collapse.

These instabilities occur at low temperatures by tuning some parameters of the system, signalling a quantum phase transition (QPT) [7]. Some of these instabilities have direct analogues in higher dimensional systems, while others may be more specific to 1D systems (and are analogues of $1 \mathrm{D}$ spin- $1 / 2$ fermionic systems $[8,9]$ ). Dilute cold gases are ideal for observing the instabilities that we predict because, in contrast to conventional solid-state materials, they are cleaner and there is a large degree of control and tunability of parameters such as particle densities and the sign and strength of the interaction.
Furthermore, diluteness makes it a priori possible to relate microscopic parameters such as scattering lengths and atomic masses to the parameters of the low-energy effective theory.

Contrary to their 3D counterparts 10, 11, 12, 13], 1D binary mixtures have only recently attracted interests [14, 15]. To our knowledge, there has been no analytic treatment of these systems within the Luttinger liquid framework. This is undertaken here using the harmonic fluid approach, which takes into account phase and density fluctuations in 1D fluids [5, [6]. We summarize our results:

Case 1.- We consider a binary mixture of two distinct atomic species (either $B+B$ or $B+F$ ) or of two internal states of the same bosonic species. When a sufficiently repulsive interaction exists between the components, the mixture will be unstable against demixing, whereas for sufficient attraction, it will collapse.

Case 2.- We consider a binary mixture in a low-density limit, when the two components are sufficiently close in density and if their sound velocities are similar, the system undergoes a QPT when there is an attraction, no matter how weak, between the two components. The resulting state exhibits a strong pairing tendency. In the case of a $B+F$ mixture, the pairing fluctuations occur at the Fermi momentum, unlike the $B+B$ case (or $F+F[\underline{8}]$ ) where they occur at zero momentum.

Model and calculations: The experimental realization of the quasi one-dimensional systems requires a tight transverse confinement [2, 3], which nowadays can be achieved in various setups 16, 17, 18]. Our discussion below will focus on the properties of homogeneous 1D systems. This should be appropriate for the central region of large harmonic 16] or square-well traps 17]. Alternatively, a toroidal trap [18 can provide the conditions for realizing a homogeneous $1 \mathrm{D}$ system. When necessary, we shall also discuss the effect of longitudinal confinement and finite size on the results to be presented below.

Our starting point will be the following Hamiltonian 
for a mixture of two $1 \mathrm{D}$ dilute gases $(\alpha, \beta=1,2)$ :

$$
\begin{aligned}
H & =\int d x \sum_{\alpha}\left[\frac{\hbar^{2}}{2 M_{\alpha}} \partial_{x} \Psi_{\alpha}^{\dagger}(x) \partial_{x} \Psi_{\alpha}(x)-\mu_{\alpha} \rho_{\alpha}(x)\right] \\
& +\frac{1}{2} \int d x \sum_{\alpha, \beta} g_{\alpha \beta} \rho_{\alpha}(x) \rho_{\beta}(x) .
\end{aligned}
$$

For bosons, the field operators, $\Psi_{\alpha}(x)$, obey $\left[\Psi_{\alpha}(x), \Psi_{\alpha}^{\dagger}\left(x^{\prime}\right)\right]=\delta\left(x-x^{\prime}\right)$, commuting otherwise. For a spin-polarized fermion, the field operator obeys anti-commuting relations: $\left\{\Psi_{2}(x), \Psi_{2}^{\dagger}\left(x^{\prime}\right)\right\}=\delta\left(x-x^{\prime}\right)$. The density operators $\rho_{\alpha}(x)=\Psi_{\alpha}^{\dagger}(x) \Psi_{\alpha}(x)$. The interatomic interaction potential is $v_{\alpha \beta}(x)=g_{\alpha \beta} \delta(x)$, with $g_{\alpha \beta}=2 \hbar \omega_{\perp} a_{\alpha \beta}$ [3] , where $a_{\alpha \beta}$ are the scattering lengths parametrizing the 3D interaction between species $\alpha$ and $\beta$, and $\omega_{\perp}$ is the transverse confinement frequency.

To study the low-energy properties of the above model, we use the harmonic fluid approach [5, 6], which employs the phase-density representation of the field operators. Phonon fields are introduced for each component: $\phi_{\alpha}(x)$ for long wavelength phase fluctuations and $\theta_{\alpha}(x)$ for long wavelength density fluctuations. It is assumed that $\partial_{x} \theta_{\alpha}(x)$ and $\partial_{x} \phi_{\alpha}(x)$ are small compared to the equilibrium densities $\rho_{0 \alpha}$. This approach treats bosons and fermions in 1D on equal footing [5, [6], and does not assume the existence of a BEC, as usual mean-field treatments do. Therefore, it can describe both systems of impenetrable bosons (e.g. the Tonks gas [4] and others [19]), and quasicondensates [2]. Introducing [5, 6] $\Psi_{\alpha}(x) \sim$ $\left[\rho_{0 \alpha}+\partial_{x} \theta_{\alpha}(x) / \pi\right]^{1 / 2} \sum_{m} e^{i m \pi \rho_{0 \alpha} x+i m \theta_{\alpha}(x)} e^{i \phi_{\alpha}(x)}$, where the sum over $m$ involves only even (odd) integers if the operator is bosonic (fermionic), and $\rho_{\alpha}=\left[\rho_{0 \alpha}+\partial_{x} \theta_{\alpha}(x) / \pi\right] \sum_{m=-\infty}^{+\infty} e^{2 i m \pi \rho_{\mathrm{o} \alpha} x+2 i m \theta_{\alpha}(x)}$, into Eq. (11) one obtains the low-energy Hamiltonian:

$$
\begin{gathered}
H_{\mathrm{eff}}=\frac{\hbar}{2 \pi} \int d x \sum_{\alpha}\left[\frac{v_{\alpha}}{K_{\alpha}}\left(\partial_{x} \theta_{\alpha}\right)^{2}+v_{\alpha} K_{\alpha}\left(\partial_{x} \phi_{\alpha}\right)^{2}\right] \\
+\frac{\hbar}{2 \pi} \int d x\left[2 \tilde{g}_{f} \partial_{x} \theta_{1} \partial_{x} \theta_{2}+\tilde{g}_{b} \cos 2\left(\theta_{1}-\theta_{2}+\pi \delta x\right)\right] .
\end{gathered}
$$

In Eq.(2), we have retained only terms which can have the most dominant effects at low energies (i.e. marginal or relevant in the renormalization group sense). Its validity is restricted to energies smaller than the chemical potential. The low-energy physics is fully characterized by the phenomenological parameters $K_{1,2}$, the sound velocities $v_{1,2}$, the couplings $\tilde{g}_{\mathrm{f}, \mathrm{b}}$, and $\delta=\rho_{01}-\rho_{02}$. For small enough $g_{12}$, it is possible to relate these phenomenological parameters to the microscopic ones because exchange and correlation effects will be of $\mathrm{O}\left(g_{12}^{2}\right)$ in $\tilde{g}_{\mathrm{f}, \mathrm{b}}$ (Otherwise, they must be extracted from numerics or experiments [6]). First notice that $v_{N \alpha}=$ $1 /\left(\hbar \pi \rho_{\mathrm{o}}^{2} \kappa_{S \alpha}\right)$, where $\kappa_{S \alpha}=\rho_{\alpha}^{-2}\left(\partial \rho_{\alpha} / \partial \mu_{\alpha}\right)$ is the compressibility [5, 6$]$. Furthermore, in homogeneous systems, Galilean invariance fixes the product $v_{J \alpha}=v_{\alpha} K_{\alpha}=$
$v_{F \alpha}=\hbar \pi \rho_{0 \alpha} / M_{\alpha}[\underline{5}$. Thus from the exact solution for a single-component 1D Bose fluid [20], $v_{N \alpha}=v_{\alpha} K_{\alpha}^{-1}=$ $v_{F \alpha}\left[1-8 \gamma_{\alpha}^{-1}+O\left(\gamma_{\alpha}^{-2}\right)\right]$ for $\gamma_{\alpha}=M_{\alpha} g_{\alpha \alpha} / \hbar^{2} \rho_{0 \alpha} \gg 1$ and $v_{N \alpha} \simeq v_{F \alpha} \gamma_{\alpha} / \pi^{2}$, for $\gamma_{\alpha} \lesssim 1$. Therefore, $1 \leq K_{\alpha}<$ $+\infty$ for bosons, $K_{\alpha}=1$ in the Tonks limit. For spinpolarized fermions, $K_{2} \simeq 1$, because the s-wave scattering length vanishes thanks to the Pauli principle, leaving the much weaker p-wave channel, which can be neglected. Finally, in the weak coupling limit, $\tilde{g}_{f} \simeq g_{12} /(\hbar \pi)$ and $\tilde{g}_{b} \simeq 4 \pi g_{12} \rho_{01} \rho_{02} / \hbar$. The sign and strength of $g_{12}$ can be controlled using a Feshbach or a confinement induced [3] resonance. Interestingly, the same Hamitonian, Eq (2), also describes a binary mixture in a 1D optical lattice 21] provided that none of the components is commensurate with the lattice periodicity. This allows for further possibilities of tuning the parameters $v_{\alpha}, K_{\alpha}$, and $g_{12}$ (e.g. the strength of $g_{12}$ can be modified by shifting relative to each other the lattices where each component hops).

The above Hamiltonian, Eq. (2), describes a rich variety of one-dimensional binary mixtures:

Case 1.- When the equilibrium densities of the two species are different, i.e. for sufficiently large $\delta=\rho_{01}-$ $\rho_{02}$, the cosine term in Eq. (2) can be neglected (see below). Thus one is left with two Luttinger liquids coupled by $\tilde{g}_{f} \partial_{x} \theta_{1} \partial_{x} \theta_{2}$. The normal modes of the system can be found from the equations of motion for $\theta_{1}(x, t)$ and $\theta_{2}(x, t)$. The phase velocity of the normal modes is:

$$
v_{ \pm}^{2}=\frac{1}{2}\left(v_{1}^{2}+v_{2}^{2}\right) \pm \frac{1}{2} \sqrt{\left(v_{1}^{2}-v_{2}^{2}\right)^{2}+4\left(v_{J 1}\right)\left(v_{J 2}\right) \tilde{g}_{f}^{2}} .
$$

Hence, the mixture will become unstable provided that

$$
\sqrt{v_{N 1} v_{N 2}}<\left|\tilde{g}_{f}\right| .
$$

For a repulsive interaction $\left(\tilde{g}_{f}>0\right)$, the two components of the mixture repel each other sufficiently strongly to demix. However, for an attractive interaction $\left(\tilde{g}_{f}<0\right)$, the system is expected to collapse.

We now examine the linear instability condition, Eq. (4), in several limiting cases. For a $B+B$ mixture in the quasicondensate regime $\left(\gamma_{1,2} \lesssim 1\right), v_{N \alpha} \simeq v_{F \alpha} \gamma_{\alpha} / \pi^{2}$ and $\tilde{g}_{f} \simeq g_{12} /(\hbar \pi)$. Then the mixture is unstable when $\sqrt{g_{11} g_{22}}<\left|g_{12}\right|$. In higher dimensional systems this condition is obtained from a mean-field theory [13], which implicitly assumes the existence of a condensate. It is therefore interesting that it also applies to $1 \mathrm{D}$ systems in the quasicondensate regime, where the phase fluctuates. Another interesting limit is a $B+F$ mixture where the boson is a quasi condensate. For the fermion, $v_{N 2} \simeq v_{F 2}=\hbar \pi \rho_{02} / M_{2}$. Hence, the system will become unstable below a critical fermion density $\rho_{02}^{\text {crit }}=M_{2} g_{12}^{2} /\left(\pi^{2} \hbar^{2} g_{11}\right)$. This agrees with the meanfield result of Das 14]. Lastly, consider a $B+F$ or a $B+B$ mixture with impenetrable boson(s) $\left(\gamma_{\alpha} \gg 1\right)$. Then Eq. (4) implies that the system becomes unstable when $\left(\rho_{01} \rho_{02}\right)<M_{1} M_{2} g_{12}^{2} /\left(\pi^{4} \hbar^{4}\right)$. 
We now briefly discuss the properties of a stable mixture. As found above, at long wavelengths there will be two branches of modes with phase velocities $v_{ \pm}$given by Eq. (3). This result is not qualitatively affected by the presence of a longitudinal confinement, though the actual energies of the modes may. More interestingly, a stable binary mixture will be a Luttinger liquid with all correlations characterized (in the thermodynamic limit and at zero temperature) by the usual power laws [5]. In a trapped system, the power laws will be accurate near the center of the trap [6]. A full discussion of these issues will be given elsewhere [21].

Case 2: When the components of the mixture are close enough in density, one can no longer neglect the cosine term in Eq. (2). To see this, consider the Tonks limit where the bosons behave as free fermions. Then this term describes a backscattering process between the fermions: A fermion of each species is excited from one Fermi point to the other, exchanging a momentum close to $2 \pi\left(\rho_{0} \pm \delta\right)$. If $\delta \ll \rho_{0}=\left(\rho_{01}+\rho_{02}\right) / 2$ then this process can occur at low energies and needs to be taken into account. To simplify our analysis, we take $\delta=0(\delta \neq 0$ will be addressed at the end). By a perturbative analysis of the free energy, we see that the correction due to this term becomes larger than the zeroth order term as $T \rightarrow 0$ for $K_{1}+K_{2}<2$. To go further, we use the renormalization group (RG) method and take $K_{1}=K_{2}=1$ and $v_{1}=v_{2}$ (We have checked that small deviations from these assumptions do not alter the physical picture qualitatively [21]). The rationale for this is that the lower bound in a boson system is $K_{\alpha}=1$, which is reached for the Tonks gas limit (i.e. by reducing the density, increasing the scattering lengths $a_{\alpha \alpha}$ or both [2]). In a $B+F$ mixture, however, the fermion already has $K_{2} \simeq 1$, and we only need to tune the boson to the Tonks limit. Furthermore, if the velocites $v_{1}$ and $v_{2}$ can be made made similar [23], then in the Tonks limit at equal densities, $v_{1}=v_{2}=v_{F}$. Thus the Hamiltonian (2) becomes $H_{\text {eff }}=H_{+}+H_{-}$, where:

$$
\begin{aligned}
H_{+} & =\frac{\hbar v_{+}}{2 \pi} \int d x\left[K_{+}^{-1}\left(\partial_{x} \theta_{+}\right)^{2}+K_{+}\left(\partial_{x} \phi_{+}\right)^{2}\right] \\
H_{-} & =\frac{\hbar v_{F}}{2 \pi} \int d x\left[\left(\partial_{x} \theta_{-}\right)^{2}+\left(\partial_{x} \phi_{-}\right)^{2}\right] \\
& +\frac{\hbar}{2 \pi} \int d x\left[\tilde{g}_{b} \cos \sqrt{8} \theta_{-}(x)-\tilde{g}_{f}\left(\partial_{x} \theta_{-}\right)^{2}\right] .
\end{aligned}
$$

The new fields $\theta_{ \pm}(x)=\left(\theta_{1}(x) \pm \theta_{2}(x)\right) / \sqrt{2}$ and $\phi_{ \pm}(x)=$ $\left(\phi_{1}(x) \pm \phi_{2}(x)\right) / \sqrt{2}$ describe the in-phase ("+") and out-of-phase ("-") density and phase fluctuations of the components of the mixture. The fact that in this limit they are decoupled is an analog of spin-charge separation, so ubiquitous in 1D Fermi systems [9, 22].

$H_{+}$has the form of a Luttinger liquid Hamiltonian characterized by the parameters $K_{+}=$ $\left[1+\tilde{g}_{f} / v_{F}\right]^{-1 / 2} \simeq\left[1+g_{12} /\left(\hbar \pi v_{F}\right)\right]^{-1 / 2}$ and $v_{+}=$ $v_{F}\left[1+\tilde{g}_{f} / v_{F}\right]^{1 / 2} \simeq v_{F}\left[1+g_{12} /\left(\hbar \pi v_{F}\right)\right]^{1 / 2}$. Hence, in- phase modes are gapless (in the thermodynamic limit).

$H_{-}$is a sine-Gordon model [9]. The relative importance of the marginal operators, the cosine term and $\left(\partial_{x} \theta_{-}\right)^{2}$, can be assessed using the RG at weak coupling, where $g_{f}=\tilde{g}_{f} / v_{F} \ll 1$ and $g_{b}=\rho_{0}^{-2} \tilde{g}_{b} / 4 \pi^{2} v_{F} \ll 1$. To second order in these couplings, the scaling flow is of Berezinskii-Kosterlitz-Thouless (BKT) type (see e.g. Refs. 7, 9]). It is interesting to note that if the sound velocities are tuned to be equal (i.e. $v_{1}=v_{2}$ ) in the limit $K_{1}=K_{2}=1$, then the RG flow proceeds along the separatrix $g=g_{f}=g_{b} \propto g_{12}$ and the spectrum of the system exhibits an enhanced SU(2) symmetry. This is not present in the microscopic Hamiltonian (1), and seems quite striking, especially for a $B+F$ mixture. Thus, for small $g_{12}>0$, the effective coupling $g$ is renormalized to zero as the temperature is decreased and the cosine term leads only to subleading corrections to the low-temperature properties: the out-of-phase modes will behave as a Luttinger liquid with $K_{-}=1$ and $v_{-}=v_{F}$. But when small $g_{12}<0$, the effective coupling $g_{b} \rightarrow+\infty$ with decreasing temperature. The exact solution of the sine-Gordon model [9] then tells us that a gap opens for the excitations of the out-of-phase mode. At weak $g \propto g_{12}$ the gap $\Delta \sim|g|^{1 / 2} e^{-1 /|g|}[7,9]$, i.e. exponentially small, and it becomes larger as $g_{12}$ increases. Hence, at low enough temperatures only in-phase modes can be excited. In a finite-size system, however, the RG flow to strong coupling will be cut-off at a length scale comparable to the size of the system. Therefore, for weak attraction one should observe that the out-of-phase modes become more "stiff" until the full gap develops.

If $v_{1} \neq v_{2}$ and/or $K_{1}, K_{2} \neq 1$ (with the differences small) the effective $\mathrm{SU}(2)$ symmetry is lost and the transition is of BKT type, the gap again being exponentially small but with a different functional form (see e.g. 7, 9]). One notable consequence of the development of the gap is that density fluctuations of both components will become correlated because only in-phase modes are allowed at low enough temperatures: specifically, the $2 \pi \rho_{0}$ part of density correlation $\left.\left\langle\rho_{\alpha}(x) \rho_{\beta}(0)\right\rangle\right|_{2 \pi \rho_{0}} \sim \cos \left(2 \pi \rho_{0}\right) x^{-K_{+}}$ for arbitrary $\alpha=\beta=1,2$ and $\alpha \neq \beta$. These correlations can be observed by exciting the modes of the system and imaging each species independently.

The aforementioned correlations are also reflected in the "pairing" correlations described by the operator $\Delta(x)=\Psi_{1}(x) \Psi_{2}(x)$. For a $B+B$ mixture, $\left\langle\Delta^{\dagger}(x) \Delta(0)\right\rangle \sim x^{-1 / K_{+}}$, which decays more slowly than the density correlation, since for $g_{12}<0, K_{+} \simeq$ $\left[1+g_{12} /\left(\hbar \pi v_{F}\right)\right]^{-1 / 2}>1$. For a $B+F$ mixture, the same correlation function has an interesting oscillation at $\pi \rho_{0}$ (rather than at $\left.2 \pi \rho_{0}\right):\left\langle\Delta^{\dagger}(x) \Delta(0)\right\rangle \sim$ $\sin \left(\pi \rho_{0} x\right) x^{-1 / K_{+}-K_{+} / 4}$. Thus, in a $B+F$ mixture, the pairing correlations will decay more slowly than the density correlations for $K_{+}>2 / \sqrt{3} \simeq 1.16$.

We now discuss the physical picture underlying the 


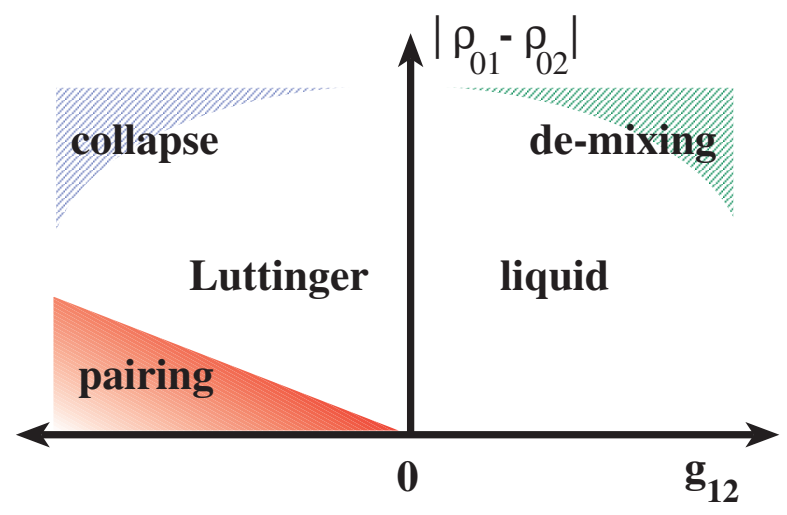

FIG. 1: Schematic phase diagram with the boson(s) in the Tonks limit. $\left|\rho_{01}-\rho_{02}\right|$ is the density difference at $g_{12}=0$.

above calculations. On intuitive grounds, an attraction between the two components of the mixture $\left(g_{12}<0\right)$ will lead to the formation of bound pairs, and might eventually lead to collapse. However, in the regime studied above, pairing takes place between hard-core bosons or a hard-core boson and a fermion, which means that as long as the repulsion between bosons of the same species is stronger, only bound pairs containing one particle of each component are possible. This picture is confirmed by considering a strong-coupling limit of the Hamiltonian (1), where first we take $g_{11}, g_{22} \rightarrow+\infty$ and then $g_{12} \rightarrow-\infty$ such that $\left|g_{12}\right| \ll g_{11}, g_{22}[24]$. Moreover, in a $B+B$ mixture pairs are also bosons, but their condensation is forbidden in $1 \mathrm{D}$ dimension by rigorous theorems 2]. In a $B+F$ mixture, the pairs are fermions, and this slightly suppresses pairing correlations relative to the $B+B$ case. We expect a smooth crossover from the weak coupling regime to the strong-coupling regime where tightly bound pairs form. As the strength of attraction between the two components increases, the size of the gap also grows thus preventing collapse (Fig. 1).

However, the gap can be destroyed when a sufficiently large difference in chemical potential leads to a density difference $|\delta|>0$. This transition is of commensurateincommensurate type [9]. Small inhomogeneities in the density due to the trap, will not have a strong effect in the pairing provided that the density difference between the two components does not become too large. Otherwise, one can expect phase segregation into pairing and nonpairing regions, with the former occurring mainly near the center of the trap.

In conclusion, we have shown that binary mixtures of 1D quantum gases exhibit some interesting instabilities. In particular, we have found a pairing instability which may be special to one dimension and should have unusual experimental signatures 21].

We acknowledge inspiring conversations with A. Nersesyan and M. Fabrizio, and useful correspondence with B.
Svistunov. A. F. H. thanks the hospitality of DIPC and a grant from EPSRC (U.K.). M. A. C. thanks Gipuzkoako Foru Aldundia for finacial support.

[1] J. R. Anglin and W. Ketterle, Nature (London) 416, 211 (2002).

[2] D. S. Petrov, G. V. Shlyapnikov, and J. T. M. Walraven, Phys. Rev Lett. 85, 3745 (2000); J. O Andersen, U. Al Khawaja, and H. T. C. Stoof, ibid. 88, 070407 (2002).

[3] M. Olshanii, Phys. Rev. Lett. 81, 938 (1998).

[4] M. D. Girardeau, J. Math. Phys. 1, 516 (1960).

[5] F. D. M. Haldane, Phys. Rev. Lett. 45, 1358 (1980); ibid 47, 1840 (1981); 48, 569 (1982) (Erratum).

[6] M. A. Cazalilla, Europhys. Lett. 59, 793 (2002); see cond-mat/0307033 for a review.

[7] S. Sachdev, Quantum Phase Transitions, Cambridge University Press (Cambridge, United Kingdom, 1999).

[8] A. Luther and V.J. Emery, Phys. Rev. Lett. 33, 589 (1974).

[9] A. O. Gogolin, A. A. Nersesyan, and A. M. Tsvelik, Bosonization and Strongly Correlated Systems, Cambridge University Press (Cambridge, United Kingdom, 1998).

[10] M. R. Matthews et al., Phys. Rev. Lett. 81, 243 (1998); D. S. Hall et al., ibid 81, 1539 (1998).

[11] A. G. Truscott et al., Science 291, 2570 (2001); F. Schreck et al., Phys. Rev. Lett. 87, 080403 (2001).

[12] T. L. Ho, Phys. Rev. Lett. 81, 742 (1998); K. Molmer, ibid 80, 1804 (1998); L. Viverit, C. J. Pethick, and H. Smith, Phys. Rev. A 61, 053605 (2000); A. Albus et al., Phys. Rev. A 65, 053607 (2002).

[13] E. Timmermans, Phys. Rev. Lett. 81, 5718 (1998).

[14] K. K. Das, Phys. Rev. Lett. 90170403 (2003).

[15] You-Quan Li et al, Europhys. Lett. 61, 368 (2003); B. Paredes and J. I. Cirac, Phys. Rev. Lett. 90, 150404 (2003); A. Albus et al., cond-mat/0304223

[16] A. Görlitz et al. Phys. Rev. Lett. 87, 130402 (2001); M. Greiner et al. ibid. 87,160405 (2001).

[17] W. Hänsel et al., Nature 413, 498 (2001).

[18] J. A. Sauer, M. D. Barrett, and M. S. Chapman, Phys. Rev. Lett. 87, 270401 (2001).

[19] M. A. Cazalilla, Phys. Rev. A 67, 053606 (2003).

[20] E. H. Lieb and W. Liniger, Phys. Rev. 130, 1605 (1963); E. H. Lieb, ibid 130, 1616 (1963).

[21] M. A. Cazalilla and A. F. Ho, unpublished.

[22] A. Recati et al., Phys. Rev. Lett. 90, 020401 (2003).

[23] On the continuum, this is the case for a $B+B$ mixture of two internal states of ${ }^{87} \mathrm{Rb}[10]$ or a $B+F$ mixture of two isotopes of $\mathrm{Li}$ 11] because their masses are equal or similar. On an optical lattice, one can adjust the lattice parameters to have $v_{1} \simeq v_{2}$ even if $M_{1} \neq M_{2}$.

[24] For a $B+B$ mixture, the limit of $g_{11}, g_{22} \rightarrow+\infty$ but finite $g_{12}$ maps to an integrable model of spinful fermions solved by C. N. Yang. For $g_{12}<0$ the fermions form bound pairs. See e.g. M. Takahashi, Thermodynamics of One-Dimensional Solvable Models, Cambridge University Press (Cambridge, 1999). 